\title{
The use of magnetic resonance mammography in women at increased risk for developing breast cancer
}

\author{
Tadeusz J. Popiela ${ }^{1}$, Wojciech Kibil ${ }^{2}$, Izabela Herman-Sucharska' ${ }^{1}$, Andrzej Urbanik ${ }^{1}$ \\ ${ }^{1}$ Chair of Radiology, Jagiellonian University Medical College, Krakow, Poland \\ 2First Chair of General, Oncological, and Gastrointestinal Surgery, Jagiellonian University Medical College, Krakow, Poland
}

Videosurgery Miniinv 2013; 8 (1): 55-62

DOI: 10.5114/wiitm.2011.31534

\begin{abstract}
Introduction: The use of conventional imaging techniques, namely mammography (MMG) and ultrasound (US), for breast cancer $(B C)$ detection in women at high risk for the disease does not bring optimal results in many cases.

Aim: The present study evaluated the effectiveness of magnetic resonance (MR) mammography (MRM) in cases where US and MMG failed to detect suspected breast lesions.

Material and methods: The study group consisted of 379 women who had had no breast pathologies detected by US and $M M G$. This group was then divided into 4 groups according to the relative risk of breast cancer development. All the women underwent MRM, and any breast pathology detected by MRM was then verified by open surgical biopsy (OSB). Results: Based on the MRM findings, 37 women with breast pathologies were identified. All detected pathologies were then classified into one of the BIRADS (Breast Imaging Reporting and Data System) categories. Of these, 33 patients underwent open surgical biopsy. There were a total of 17 benign and 16 malignant breast pathologies that were not visualized by US and MMG. The types of malignancies found, in order of their frequency, were as follows: invasive ductal carcinoma (11 cases), ductal carcinoma in situ (2 cases), invasive lobular carcinoma (2 cases), and lobular carcinoma in situ (1 case). An analysis of MRM effectiveness in detecting BC showed $93.7 \%$ sensitivity and $64.71 \%$ specificity.

Conclusions: All women with a $20 \%$ or greater lifetime risk of developing BC should undergo annual MRM as a diagnostic adjunct to US and MMG.
\end{abstract}

Key words: breast cancer, magnetic resonance mammography, open surgical biopsy.

\section{Introduction}

A family history of breast cancer has long been associated with environmental factors, but is now attributed chiefly to genetic factors. Both a high incidence of hereditary breast cancer and a family history of pathogenic mutations that predispose a woman to developing the disease significantly increase the risk for breast cancer even at a young age. Strategies for reducing the risk of breast cancer include bilater- al mastectomy, bilateral ovariectomy, and chemoprevention such as the use of tamoxifen.

These radical procedures are increasingly being replaced by the use of diagnostic imaging techniques in order to increase the chances of early detection of breast cancer and to maximize curative treatment.

The conventional imaging techniques used so far in women at increased risk for breast cancer, such as mammography (MMG) and ultrasound (US), have not yielded optimal results, and thus the disease is still 
generally detected at a stage that does not allow for effective treatment. At the same time, substantial progress in improving magnetic resonance mammography (MRM) technique has been made over the last decade; nevertheless, the high cost of this examination, together with its limited accessibility, make its routine use untenable. Despite this, MRM is being increasingly used as a diagnostic adjunct to MMG and US in cases where there is diagnostic uncertainty.

\section{Aim}

This study evaluates the effectiveness of MRM as a diagnostic tool complementary to MMG and US for women at increased risk for developing breast cancer.

\section{Material and methods}

Genetic testing was performed on 402 women, aged 17-78 (mean age: 48.9 years), in collaboration with the International Hereditary Cancer Center (IHCC) of the Pomerania Medical University [Collaboration group consisted of Prof. J. Lubiński, Dr T. Byrski, and L. Romańska].

The tests were performed in all women who had developed breast or ovarian cancer or had a family history that included at least one relative with documented breast cancer before the age of 50 years or one relative regardless of age with ovarian cancer. The tests were done in two phases. The patients first received complete information regarding the aims, rules, and the expected results of the study and were asked to express their informed consent. They then completed questionnaires enquiring about all known cases of cancer in relatives of first or greater than first degree of relationship, including the year of disease onset, and if applicable, the year of death from the disease. Additionally, in each case a family tree was constructed that took into consideration firstand second-degree relatives and the complete family history with regard to cancer. At the end of this first phase, 5-6 $\mathrm{ml}$ venous blood samples were drawn from the patients for genetic testing.

In the second phase, DNA testing was done in the Laboratory of the IHCC, and all results, including data from the questionnaires and family interviews, were carefully analyzed.

Approximately 1 month prior to obtaining the DNA results, the women were subjected to:

1. Clinical breast examination.
2. Breast US using a Hitachi 740 unit with $7.5 \mathrm{MHz}$ linear probe or Voluson 730 Pro unit with 6-12 MHz linear probe.

3. And for those over 35 years of age, MMG (provided it had not already been performed within the last 12 months).

All breast lesions were classified according to MMG BIRADS categories [1].

Of the 402 women subjected to the examinations, a group of 379 (94.28\%), aged 17-77 years (mean age: 47.54 years), who either had had no focal lesions detected (BIRADS 1) or had only benign lesions (BIRADS 2), was identified.

All the women underwent MRM. Furthermore, they were divided into 4 subgroups based on the genetic testing results as follows:

- Group I - 84 women (22.16\%), aged 26-66 years (mean: 49.12 years), who carried pathogenic mutations: BRCA1 - 79 (94.05\%), BRCA2 - 3 (3.57\%), CHECK2 - 2 (2.38\%). Because of genetic mutations, the risk of developing breast cancer in this group was more than $20 \%$ [2].

- Group II - 39 women (10.29\%), aged 17-72 years (mean: 41.18 years), with confirmed hereditary breast cancer - site specific (HBC-ss) or hereditary breast-ovarian cancer (HBOC) syndromes. Regardless of the number of breast cancer cases in the family, the risk of developing breast cancer for these women was greater than $20 \%$ [2].

- Group III - 32 women (8.44\%), aged 39-77 years (mean: 52.32 years), who had had mastectomy or breast-conserving therapy for breast cancer. In these women no genetic mutations were found that would predispose them to developing breast cancer. Regardless of the type of operation performed, the estimated mean risk for these patients of developing breast cancer in the bilateral breast, based on the Claus risk tables, is greater than 4\% [3]. After breast-conserving therapy, the risk of local recurrence within 10 years of the surgery is approximately $7 \%[3,4]$.

- Group IV - 224 women (59.10\%), aged $19-52$ years (mean: 47.54 years), who reported single breast or ovarian cancer cases in a family member of first or greater than first degree of relationship. However, the number of cancers and the age of the patient at the time of the cancer onset did not allow for a diagnosis of HBC-ss or HBOC syndromes.

According to the Claus model, the estimated lifetime risk for breast cancer for these women based on the number of cases, age, and the degree of relationship is less than $20 \%[3,4]$. 
Magnetic resonance mammography was performed on the menstruating women between the $6^{\text {th }}$ and $17^{\text {th }}$ days of the menstrual cycle, and for all patients any gonadotropic hormonal administration (contraceptives, hormone replacement therapy, etc.) was withdrawn at least 1 month prior to the scheduled MRM.

Magnetic resonance mammography was performed using the GEMS Signa Excite 1.5T system with INVIVO 4-channel double breast coil with the patient in the prone position. In unenhanced MR we used frFSE (fast relaxation fast spin echo) T2, FSE T1 and frFSE T2 sequences with fat saturation in the axial view, and frFSE T2 in the sagittal view. Additionally, in the coronal view, the SE (spin echo) T1 sequence was used to evaluate axillary lymph nodes before contrast administration. Contrast-enhanced MR was performed in the 3D GRE (gradient recalled echo) T1 DYN sequence with 6-fold repetition after contrast administration, with the field of view covering the entire breast and the acquisition time not exceeding $1 \mathrm{~min}$. The SE T1 sequence was repeated in the coronal view in order to assess axillary lymph nodes after contrast administration. We used a $512 \times 512$ matrix with a rectangular field of view $24 \mathrm{~cm} \times 38 \mathrm{~cm}$, providing $4 \mathrm{~mm}$ thickness slices, and phase encoding along the body axis. In all patients, Multihance contrast agent was administered intravenously in a dose of $0.1 \mathrm{mmol} /$ $\mathrm{l} / \mathrm{kg}$ body mass.

Breast lesions visualized by MRM were classified into one of the MR BIRADS categories developed by the American College of Radiology [5].

Those patients whose breast lesions were not visualized by US and MMG, but were detected by MRM, were qualified for a second-look US and their mammograms were analyzed again. All patients with lesions identified in such a way were referred for open surgical biopsy after introducing the localization needle under US or MMG guidance.

All lesions that continued to be visible only by $M R M$, regardless of their BIRADS type, were subjected to MR-guided needle localization open breast biopsy (Figure 1).

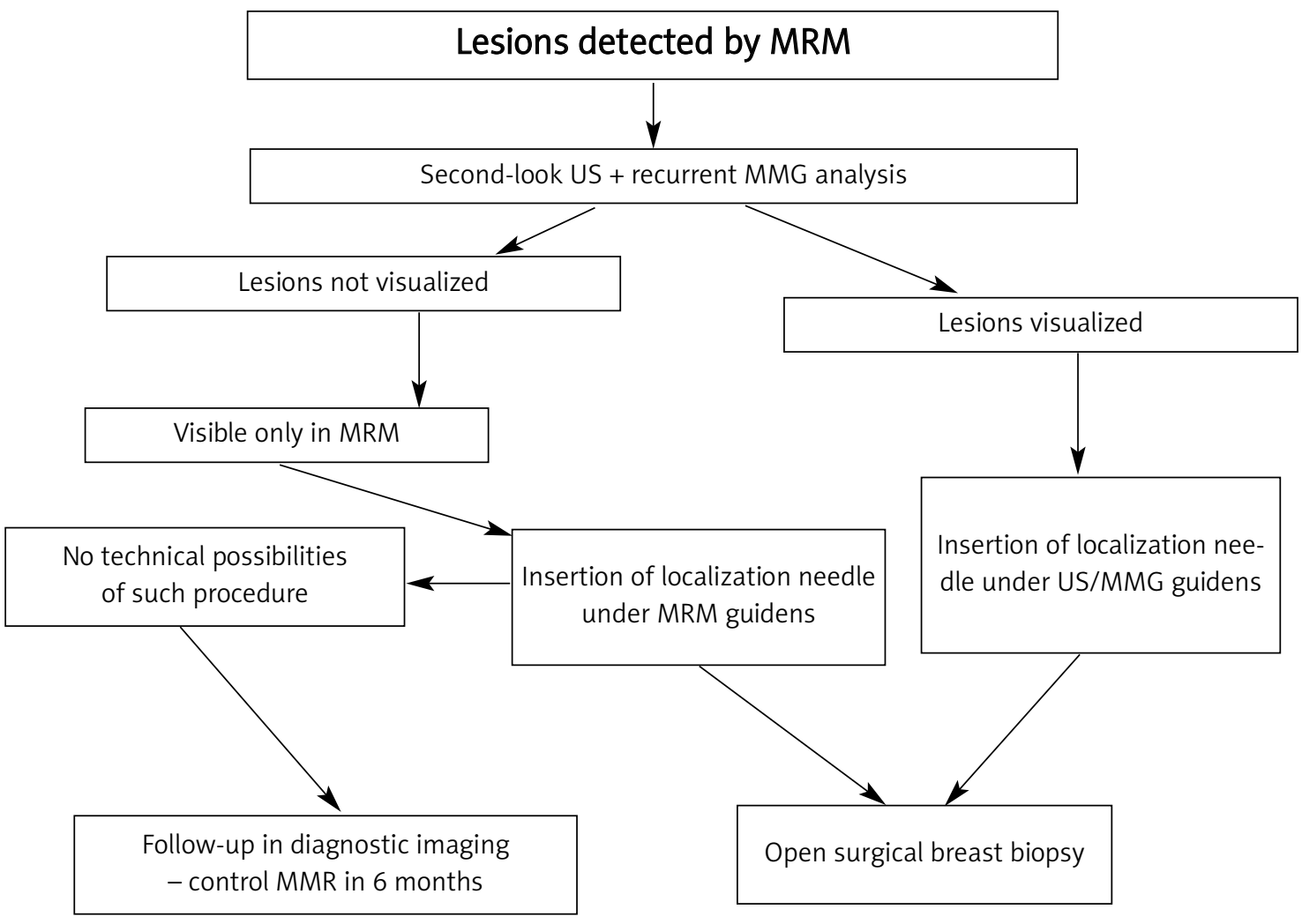

Figure 1. Diagnostic procedures used in patients with lesions detected by MRM 


\section{Statistical analysis}

For the purpose of statistical analysis we selected a group of women in whom breast MR revealed lesions that were not visualized in the previous examinations. Chi square test and Fisher's exact test were used for the analysis of statistical significance between histopathological findings (obtained from the open surgical biopsies) and BIRADS categories based on the diagnostic breast MR diagnosis. Breast cancer true positive and true negative rates were calculated for the individual BIRADS categories to estimate sensitivity and specificity of breast MR in detecting cancer. The false negative rate was calculated for the BIRADS categories 2 and 3 , and the false positive rate for the BIRADS categories 4 and 5 . We also estimated the positive predictive value (PPV) for breast cancer in lesions categorized as BIRADS 4 and 5, and the negative predictive value (NPV) in lesions classified as BIRADS category 2 and 3. The number of breast cancer cases was estimated for the individual 4 groups of patients enrolled in the study. All calculations were done using SPSS 14 statistical software.

\section{Results}

Of the 380 examined patients, 37 women (9.74\%) were identified with breast pathologies detected by MRM that were not visualized by previous US and MMG.

In 1 of these patients two breast pathologies were found over a 3-year period, and we thus considered this particular case twice in the analysis. In the remaining 343 women (90.26\%), MRM did not reveal any lesions that had not also been visualized by US and MMG. The 37 women were then classified into the appropriate MR BIRADS categories based on their MRM findings (Table I).

Table I. Number of patients with lesions detected by MRM not visualized by earlier US/MMG, classified into MR BIRADS categories

\begin{tabular}{|ccc|}
\hline BIRADS & No. of patients & Percentage \\
\hline 2 & 8 & 2.11 \\
\hline 3 & 8 & 2.11 \\
\hline 4 & 13 & 3.42 \\
\hline 5 & 8 & 2.11 \\
\hline Total & 37 & 9.75 \\
\hline
\end{tabular}

Eight women $(2.11 \%)$ had lesions interpreted as being benign and hence were classified in group II BIRADS. In the next 8 patients (2.11\%), MRM findings were indeterminate, and they were therefore classified in group III BIRADS. Thirteen (3.42\%) and $8(2.11 \%)$ patients were classified in groups IV and V BIRADS respectively, indicating a high or very high risk of breast cancer.

All 37 women with breast lesions detected by MRM were subjected to a second-look US, and their mammograms were analyzed again. In 4 (10.81\%) of the women, the findings corresponded to the MRM diagnosis and these women were subjected to either US-guided needle localization open surgical breast biopsy (OSBB) (3 patients) or mammography-guided needle localization OSB (1 patient).

In the remaining 33 women (89.19\%), secondlook examinations did not reveal lesions that were visualized by MRM, and these patients were qualified for OSB following MRM-guided localization of the lesion.

In 4 (12.12\%) of these women, however, a biopsy could not be performed. In two women there were technical problems with reaching the lesion by the localization needle tip, and in another two, the lesions were not visualized in the preceding OSB second MRM, which precluded the use of the localization needle. These patients qualified for routine follow-up visualizing examinations.

Finally, OSB was performed on 33 women, including 29 who had had breast lesions visualized by MRM alone, and 4 who had had pathologies visualized by either MMG or US examination performed after MRM.

The open breast biopsy histopathological findings were correlated with MR BIRADS categories as follows:

- in BIRADS 2 group - 7 (100\%) benign breast lesions, - in BIRADS 3 group - 1 (16.67\%) invasive ductal cancer and 5 benign lesions (83.33\%),

- in BIRADS 4 group - 7 (53.84\%) malignant lesions: 2 ductal carcinomas in situ (15.38\%), 4 (30.77\%) invasive ductal carcinomas, 1 invasive lobular carcinoma (7.69\%), and 5 (46.16\%) benign lesions,

- BIRADS 5 group - 8 cancers (100\%): 6 invasive ductal carcinomas, 1 lobular carcinoma in situ and 1 invasive lobular carcinoma (Table II).

Altogether, 17 benign and 16 cancerous lesions were visualized solely by MRM. The types of cancerous lesions in the order of their frequency were as 
Table II. Histopathological findings after OSB compared to MR BIRADS groups

\begin{tabular}{|c|c|c|c|c|c|c|c|c|c|c|c|}
\hline & & \multicolumn{8}{|c|}{ MR BIRADS groups } & \multicolumn{2}{|c|}{ Total } \\
\hline & & \multicolumn{2}{|c|}{2} & \multicolumn{2}{|r|}{3} & \multicolumn{2}{|c|}{4} & \multicolumn{2}{|c|}{5} & \multirow[b]{2}{*}{$n$} & \multirow[b]{2}{*}{$\%$} \\
\hline & & $n$ & $\%$ & $n$ & $\%$ & $n$ & $\%$ & $n$ & $\%$ & & \\
\hline \multirow[t]{8}{*}{ Hist-path after OSB } & 1 & 1.00 & 16.67 & 0.00 & 0.00 & 1.00 & 7.70 & 0.00 & 0.00 & 2.00 & 6.06 \\
\hline & 2 & 0.00 & 0.00 & 0.00 & 0.00 & 2.00 & 15.38 & 0.00 & 0.00 & 2.00 & 6.06 \\
\hline & 3 & 0.00 & 0.00 & 1.00 & 16.67 & 4.00 & 30.76 & 6.00 & 75.00 & 11.00 & 33.33 \\
\hline & 4 & 0.00 & 0.00 & 0.00 & 0.00 & 0.00 & 0.00 & 1.00 & 12.50 & 1.00 & 3.03 \\
\hline & 5 & 0.00 & 0.00 & 0.00 & 0.00 & 1.00 & 7.70 & 1.00 & 12.50 & 2.00 & 6.06 \\
\hline & 6 & 0.00 & 0.00 & 0.00 & 0.00 & 1.00 & 7.70 & 0.00 & 0.00 & 1.00 & 3.03 \\
\hline & 7 & 5.00 & 83.33 & 3.00 & 50.00 & 2.00 & 15.38 & 0.00 & 0.00 & 10.00 & 30.30 \\
\hline & 8 & 0.00 & 0.00 & 2.00 & 33.33 & 2.00 & 15.38 & 0.00 & 0.00 & 4.00 & 12.12 \\
\hline Total & & 6.00 & 100.00 & 6.00 & 100.00 & 13.00 & 100.00 & 8.00 & 100.00 & 33.00 & 100.00 \\
\hline
\end{tabular}

$\left(\chi^{2}(21)=31.54, p<0.35\right.$, Fisher test $\left.=27.67, p<0.12\right)$

$n$-number of patients, Histopathological findings after OSB: 1 - fibro-cystic lesions, 2 - ductal carcinoma in situ, 3 - invasive ductal carcinoma, 4 - lobular carcinoma in situ, 5 - invasive lobular carcinoma, 6-lymphatic nodule, 7 - other benign lesions (apocrine metaplasia, non-atypical hyperplasias), 8 - intraductal papilloma

follows: invasive ductal carcinoma (11 cases), ductal carcinoma in situ ( 2 cases), invasive lobular carcinoma (also 2), and lobular carcinoma in situ (1 case).

Based on the above findings (especially the BIRADS results), the sensitivity of breast MRM in detecting malignant lesions was $93.75 \%$ and specificity $64.71 \%$.

The positive predictive value of MRM in detecting cancer lesions was $71.43 \%$ and the negative predictive value was $91.67 \%$. Thus, the probability of developing breast cancer in women classified as BIRADS 4 or 5 was more than $70 \%$, while the probability of breast lesions being benign in women classified as BIRADS 2 and 3 was more than 90\%. Lastly, there was one false negative result in the BIRADS 3 category, and 6 false positive results in BIRADS 4.

We analyzed the incidence of cancer in 4 groups of women divided according to their relative risk for developing breast cancer. There were 12 (75\%) breast cancer cases in women with documented HBC-ss and $\mathrm{HBOC}$ in group II, $3(18.75 \%)$ cases in genetic mutation carriers in group I, and $1(6.25 \%)$ in a woman with a family history of breast cancer from group IV. There were no cases of breast cancer in group III in those patients who had had operations for breast cancers (Table III).

All 343 women with benign lesions detected by US or MMG and confirmed by MRM were offered annual clinical breast examinations, US, MMG, and MRM. The only exceptions were those women under 30 years of age on whom MMG had not been performed. The follow-up time is currently 3.5 years.

In total, 229 women (67\%) were followed up according to the protocol. In none of these did MRM detect any breast lesions other than those diagnosed by US and MMG.

Table III. Number of detected breast cancers in the individual risk groups

\begin{tabular}{|ccc|}
\hline Groups & No. of breast cancers & Percentage \\
\hline I & 3 & 18.75 \\
\hline II & 12 & 75.0 \\
\hline III & 0 & 0 \\
\hline IV & 1 & 6.25 \\
\hline Total & 167 & 100 \\
\hline
\end{tabular}




\section{Discussion}

A standard diagnostic protocol used for women at increased risk for developing breast cancer in Poland covers breast US in patients over 25 years of age and MMG alternately with US biannually when patients reach the age of 35 . In patients with the highest risk for breast cancer, however, this strategy is not enough to significantly reduce the cancer risk. Preventive administration of tamoxifen and even bilateral mastectomy with simultaneous breast prosthesis implantation are also recommended [6], but these approaches are not satisfactory for some of the women from the high risk group; for them still other measures with an increased capability of detecting breast cancer need to be searched for.

Magnetic resonance mammography is not routinely performed due to the limited access and high costs of the examination. Nevertheless, its greater effectiveness in detecting breast cancer when compared with conventional visualizing methods is increasingly underlined. Magnetic resonance mammography is highly sensitive in detecting occult carcinoma which produces distant metastases [7].

In studies conducted since 2000, breast cancer detectability in asymptomatic women at increased risk for breast cancer has ranged from $4.6 \%$ to $7.6 \%$ [8-10]. These findings correspond to our observations, where 16 cases of breast cancer were detected; this number represents $4.2 \%$ of the total number of patients involved.

The results of our study have shown that all lesions classified as BIRADS 2 were benign. A single case of a false negative diagnosis occurred in BIRADS 3 category, which is on the borderline, and regardless, the patient was qualified to have a control MRM in 6 months. The final diagnosis in this case was lowdifferentiated ductal carcinoma in situ. Moreover, according to the current literature, a false negative interpretation of the MRM results is quite common in this type of cancer $[7,11]$. In the BIRADS 4 category there were 6 false positive results, which correlated with the lower specificity of the MRM reported by other authors $[8,9]$. All the lesions were accurately classified as BIRADS 5 and did turn out to be breast cancer.

The positive predictive value of MRM, which is $71.43 \%$ in the range of $17-88 \%$, was estimated in studies on the effectiveness of screening MRM in women with a high risk of developing breast cancer $[8-10,12]$.
Magnetic resonance-guided needle localization open breast biopsy was performed on 29 women involved in this study. There were 4 other women with breast abnormalities detected by the secondlook US and repeated analysis of mammograms; on these women OSB was performed after inserting the localization needle guided by one of the imaging methods. Such an approach allows the patient to avoid a second MRM (for lesion localization) and so simplifies the diagnostic procedure. We should remember that these lesions could not be detected without MRM diagnosis. A similar approach was introduced in 2000; however, instead of OSB, a fineneedle aspiration biopsy was used [13]. In successive years this technique was abandoned for patients at increased risk for developing breast cancer because of the high number of false negative results [14].

In 4 patients open breast biopsy was unsuccessful because of technical problems. In 2 patients the lesions were localized deep in the upper inside quadrant of the breast and were therefore inaccessible for the guiding coaxial needle. Large breast size coupled with only lateral access for needle insertion made this type of lesion inaccessible even for a $15 \mathrm{~cm}$-long guiding needle. The use of a bilateral coil is thus recommended for MR-guided interventions; this allows direct access to the outside as well as inside quadrants of the breast [14].

In the other 2 patients, OSB could not be performed because a second MRM (performed for lesion localization) did not visualize the lesion. As has been reported in different studies $[10,14]$, this problem occurs in $5-8 \%$ of all MRM examinations and may be influenced by the activity of gonadotropic hormones. Thus withdrawing hormone therapy at least 1 month prior to the examination and performing MRM between the $5^{\text {th }}$ and $17^{\text {th }}$ days of the menstruation cycle are legitimate recommendations. Another reason OSB cannot be successfully performed may have to do with breast compression that is too dense for the insertion of the localization needle during the MRM. Consequently, some investigators recommend a control MRM without breast compression $24 \mathrm{~h}$ prior to the examination. Additionally, according to recently published observations, the lesions may not appear during second MRM because of being transient breast inflammation foci [14].

The majority of the cancer cases were detected in groups I and II, where the risk of developing breast cancer was more than $20 \%$. As many as $75 \%$ of the 
breast cancers were found in women with documented HBC-sS or HBOC (group II). Surprisingly, these women were not carriers of BRCA1 mutations; however, they were from families in which individuals from multiple generations had had cancer, often of early onset. In our study we followed the guidelines of the American Cancer Society from 2007 which recommended the use of MRM in women with a lifetime risk of breast cancer greater than 20\% [2]. At the same time, MRM was not recommended for women with a less than $20 \%$ risk of the disease, such as our patients from groups III and IV, in only one of whom breast cancer was actually found.

Thus there are no clear indications for performing MRM on a regular basis in women with a hereditary risk of breast cancer without a documented diagnosis of mutation or HBC-ss or HBOC syndromes. The situation is similar for women after mastectomy; in such cases the risk of bilateral breast cancer is $0.5-1.0 \%$ annually, or $5-10 \%$ more than 10 years after the initial diagnosis [2]. For women in each of the above situations, the risk of developing breast cancer is less than $20 \%$.

In studies on the effectiveness of MRM in women at high risk for developing breast cancer, the sensitivity of the examination has been shown to be in the range of $77-100 \%$, and the specificity in the range of $81-90 \%$ [2, 12, 15]. In our particular study, MRM sensitivity was about $94 \%$ and specificity $65 \%$, i.e., lower than in the reported observations.

This may reflect the interpretation of BIRADS 4 abnormalities as cancer for the purpose of statistical analysis, even though such abnormalities would only be considered suspicious lesions according to the BIRADS definition. All lesions with definite features of $B C$ are classified as BIRADS 5 .

The reduced specificity of MRM relative to its sensitivity may yield more false positive results than other visualizing methods. This may lead to having to qualify more patients for extended diagnostic procedures, second-look MRM, and open breast biopsies. It seems that MR-guided biopsy may be a solution to this problem and may effectively increase the specificity of MRM. The preliminary results of MRM-guided needle biopsies are comparable to those obtained from OSB. The main advantage of the former method is that it is less invasive, but its limited availability as a highly specialized procedure constitutes a major drawback.

\section{Conclusions}

All women who are carriers of BRCA1 and BRCA2 mutations and have documented $\mathrm{HBC}$-ss and $\mathrm{HBOC}$ syndromes should undergo MRM annually as an adjunct to US and MMG. Second-look US and analysis of mammograms should be done in all cases of suspected focal lesions detected by MRM and not visualized by conventional methods. This allows for the insertion of the localization needle under the control of US or MMG and thus avoids the relatively difficult and time-consuming MRM-guided localizations. All external factors that have an effect on MRM findings should be eliminated, such as hormone replacement therapy, inappropriate day of the menstruation cycle for performing the examination, and excessive breast compression. Studies should continue to be done to improve MRM specificity.

\section{References}

1. American College of Radiology. Breast Imaging Reporting and Data System (BIRADS), 3rd ed. Reston, VA: American College of Radiology 1989.

2. Saslow D, Boetes C, Burke W, et al. American Cancer Society Guidelines for breast screening with MRI as an adjunct to mammography. A Cancer J Clin 2007; 57: 75-89.

3. Claus EB. Risk model to counsel women for breast and ovarian cancer: a guide for clinicians. Fam Cancer 2001; 1: 197-206.

4. Sanghani M, Balk E, Cady B. Predicting the risk of local recurrence in patients with breast cancer: an approach to a new computer-based predictive tool. Am J Clin Oncol 2007; 30: 473-80.

5. Erguvan-Dogan B, Whitman GJ, Kushwaha AC, et al. BI-RADSMRI: a primer. Am J Roentgenol 2006; 187: 152-60.

6. Hooks MA. Breast cancer: risk assessment and prevention. South Med J 2010; 103: 333-8.

7. Kuhl CK, Lieberman S, Sella T, et al. Breast magnetic resonance imaging characteristics in women with occult primary breast carcinoma. Isr Med Assoc J 2008; 20: 448-52.

8. Kuhl CK, Schmutzler RK, Leutner CC, et al. Breast MR imaging screening in 192 women proved or suspected to be carriers of a breast cancer susceptibility gene: preliminary results. Radiology 2000; 215: 267-79.

9. Lehman CD, Isaacs C, Schnall MD, et al. Cancer yield of mammography, MR, and US in high-risk women: prospective multiinstitution breast cancer screening study. Radiology 2007; 244: 381-8.

10. Morris EA, Liberman L, Ballon DJ, et al. MRI of occult breast carcinoma in a high-risk population. Am J Roentgenol 2003; 181: 619-26.

11. Zulani C, Francescutti GE, Londero V, et al. Ductal carcinoma in situ: is there a role for MRI? J Exp Clin Cancer Res 2002; 21: 89-95.

12. Leach M. Screening with magnetic resonance imaging and mammography of UK population at high familial risk of breast 
cancer: a prospective multicentric cohort study (MARIBS). Lancet 2005; 365: 1769-78.

13. Obdeijn IM, Brouwers-Kuyper EM, Tilanus-Linthorst MM, et al. MR imaging guided sonography followed by fine-needle aspiration cytology in occult carcinoma of the breast. Am J Roentgenol 2000; 174: 1079-84.

14. Viehweg P, Bernerth T, Heinig A, et al. MR-guided intervention in women at high hereditary risk of breast cancer due to both family and personal history of breast cancer. Breast J 2006; 12: 549-58.

15. Sardanelli F, Podo F. Breast MR imaging in women at high-risk of breast cancer. Is something changing in early breast cancer detection? Eur Radiol 2007; 17: 873-87.

Received: 30.04.2012, revised: 30.07.2012, accepted: 28.08.2012 